\title{
ON A FAMILY OF SURFACES OF REVOLUTION OF FINITE CHEN-TYPE*
}

\author{
To the memory of Prof. I. Rozas
}

\author{
J. Arroyo, O. J. Garay and J. J. Mencía
}

\begin{abstract}
We study Chen-finite type surfaces of revolution in $E^{3}$ which contain two affine circles through each point. First, we prove that they can be obtained by revolving an ellipse on a suitable axis and then we show that only the 2-dimensional sphere is of finite type.
\end{abstract}

\section{Introduction}

Euclidean submanifolds of finite type were introduced by B. Y. Chen in the late seventies and it has been a topic of active research since then (for details see [3] [4]). An Euclidean submanifold is said to be of Chen finite type if its coordinate functions are a finite sum of eigenfunctions of its Laplacian, [3]. Compact Euclidean submanifolds are characterized by both a polynomial criterium and also by satisfying a variational principle, [4]. B. Y. Chen posed in [5] [6] the problem of classifying the finite type surfaces in the 3-dimensional Euclidean space $E^{3}$. In fact the only known finite Chen-type surfaces in $E^{3}$ are portions of spheres, circular cylinders and of minimal surfaces and it was B. Y. Chen who made in [6] the following conjecture.

CONJECTURE. The only compact surfaces of finite type in $E^{3}$ are the spheres.

The conjecture is still unsolved but it has been confirmed by different authors by proving that finite type tubes, finite type ruled surfaces, finite type quadrics, finite type cones and finite type cyclides of Dupin are surfaces of the only known examples in $E^{3}$, [4]. However, for another classical family of surfaces in $E^{3}$, the surfaces of revolution, the classification of its finite type members is not known yet. Particular cases of this problem were consider in [7], [8] and [9]. In [7] two

* Partially supported by a grant of Gobierno Vasco PI 95/95

Received November 11, 1997; revised February 4, 1998. 
families of finite type surfaces of revolution with generating curve satisfying certain algebraic conditions are classified. In [8] the conjecture was confirmed for surfaces of revolution with finite type coordinate functions. Finally in [9], authors gave the classification of surfaces of revolution with constant mean curvature.

Our purpose here is to classify a family $\mathfrak{F}$ of compact finite type surfaces of revolution $M^{2}$ which is determined by satisfying the following geometric property:

(P) There are at least two affine circles (ellipses) contained in $M^{2} \in \mathfrak{F}$ through each point $p \in M^{2}$.

As a motivation for the study of the above property we remind that in 1822 C. Dupin defined a cyclide to be a surface $M^{2}$ in $E^{3}$ which is the envelope of a family of spheres tangent to three fixed spheres. All compact cyclides of Dupin in $E^{3}$ can be obtained by inversion of a torus of revolution and, therefore, they contain four metric circles through each point [2]. In $1980 \mathrm{R}$. Blum gave an example of compact cyclides with 4, 5 and 6 metric circles through each point [1] and $\mathrm{K}$. Ogiue and $\mathrm{N}$. Takeuchi [10] proved that a compact surface of revolution which contains at least two metric circles through each point, is a Hulahoop surface. Hulahoop surfaces have 4, 5 or infinitively many metric circles through each point.

In order to achieve our goal, we begin with defining the elliptical hulahoop surfaces as those surfaces of $E^{3}$ obtained by revolving an ellipse around a suitable axis. We first prove

Proposition 1. Let $M^{2}$ be a compact surface of revolution in $E^{3}$ which contains at least two ellipses through each point, then it is an elliptic hulahoop surface.

This result is analogous to the one proved in [10] for metric circles, but their method can not be applied here so that we need to furnish a different argument. Note also that our family includes the hulahoop surfaces of [10]. Then we will prove

Proposition 2. The only elliptic hulahoop surface of finite type is the sphere.

As a consequence

COROLlARY 1. The only compact finite type surface of revolution which satisfies $(\mathbf{P})$ is the sphere.

This confirms Chen's conjecture. 


\section{Preliminaries}

Let $x: M^{n} \longrightarrow E^{m}$ be an isometric immersion of a compact Riemannian manifold into the $m$-dimensional Euclidean space. The inmersion $\left(M^{n}, x\right)$ is said to be of finite Chen-type $k$ if the position vector $x$ admits the following spectral decomposition

$$
\boldsymbol{x}=\boldsymbol{x}_{0}+\sum_{t=1}^{k} \boldsymbol{x}_{\boldsymbol{t}}
$$

where $\boldsymbol{x}_{t}$ are $E^{m}$-valued eigenfunctions of the Laplacian of $\left(M^{n}, \boldsymbol{x}\right): \Delta \boldsymbol{x}_{t}=\lambda_{t} \boldsymbol{x}_{t}$.

The following minimal polynomial criterion is a useful tool to decide whether a submanifold is of finite Chen-type [3].

Proposition 3. An isometric inmersion $\left(M^{n}, x\right)$ of a compact Riemannian manifold in $E^{m}$ is of finite Chen-type, if and only if, there exists a nontrivial polynomial $Q(t)$ such that $Q(\Delta)\left(x-x_{0}\right)=0$, where $\Delta$ is the Laplacian of $\left(M^{n}, x\right)$.

Now, we want to define the elliptic hulahoop surfaces of $E^{3}$. These are surfaces of revolution which are obtained by revolving an ellipse around an axis which is not perpendicular to the plane containing the ellipse. They can be obtained in the following way: Choose the $x, y, z$ axes so that the first two are parallel to the axes of the ellipse $E(a, b, r, s) r, s>0$ given by

$$
\frac{(x-a)^{2}}{r^{2}}+\frac{(y-b)^{2}}{s^{2}}=1
$$

which is supposed to be contained in the $x y$-plane. Rotate the ellipse around its centre by an angle $\beta \in[0, \pi / 2]$ and denote by $E(a, b, r, s, \beta)$ the resulting ellipse. Finally we denote by $E(a, b, r, s, \beta, \alpha)$ the ellipse obtained by tilting $E(a, b, r, s, \beta)$ around a diameter parallel to the $x$-axis by an angle $\alpha \in[0, \pi]$. It can be easily checked that the final ellipse is parametrized by

$$
\left\{\begin{array}{l}
x=a+r \cos \beta \cos \theta-s \sin \beta \sin \theta \\
y=b+\cos \alpha(r \sin \beta \cos \theta+s \cos \beta \sin \theta) \\
z=\sin \alpha(r \sin \beta \cos \theta+s \cos \beta \sin \theta)
\end{array}\right.
$$

with $\theta \in[0,2 \pi]$.

Now let $H(a, b, r, s, \beta, \alpha)$ be the surface which is obtained by revolving $E(a, b, r, s, \beta, \alpha)$ around the $z$-axis. Then one can check by lengthly computation that $H$ is a regular surface, if and only if, either

(i) $0 \neq \alpha \neq \pi / 2$,

$0 \neq b^{2} d^{2}+a^{2} \cos ^{2} \alpha c^{2}+2 a b e \cos \alpha-r^{2} s^{2} \cos ^{2} \alpha$,

$0>e^{2}-a^{2} c^{2}$ or 
(ii) $\alpha=\pi / 2, b \neq 0$,

$0>e^{2}-a^{2} c^{2}$ or

(iii) $\alpha=\pi / 2, b=0$,

$d^{2}<a^{2}$ or

(iv) $\alpha=\pi / 2, a=b=0, r=s$ or

(v) $\alpha=\pi / 2, a=b=0, \beta=0$

where $d^{2}=r^{2} \cos ^{2} \beta+s^{2} \sin ^{2} \beta, c^{2}=s^{2} \cos ^{2} \beta+r^{2} \sin ^{2} \beta$ and $e=\left(s^{2}-r^{2}\right) \sin \beta \cos \beta$.

\section{Proofs of the results}

First we give:

Proof of Proposition 1. Since it is a compact surface of revolution, $M^{2}$ must be either a topological sphere or a topological torus.

First, we consider the simply connected case. Let $p_{0}$ be any point of $M^{2}$. Since there are two ellipses through $p_{0}$, at least one of them, $E_{0}$, is not a latitude. If the latitude $C_{0}$ through $p_{0}$ is not transversal to $E_{0}$, we can take a point on $E_{0}, p_{1}$, as close to $p_{0}$ as necessary, so that the latitude $C_{1}$ through $p_{1}$ cuts $E_{0}$ transversally. Take $p_{2}=E_{0} \cap C_{1}-\left\{p_{1}\right\}$. Let $\Pi$ be the perpendicular bisector of the segment $\overline{p_{1} p_{2}}$. Since $\overline{p_{1} p_{2}}$ forms a chord of $C_{1}, \Pi$ must contain the axis of revolution. Thus we can consider $\gamma=\Pi \cap M^{2}$ as the plane generating curve of $M^{2}$. Let us denote by $N$ the part of $M^{2}$ which is generated by the points of $E_{0}$. Clearly $\Pi \cap N$ is a closed segment $\beta$ of $\gamma$. By regularity, one can see that $\beta$ must be symmetric with respect to $\Pi$ and then it can be checked by direct computation that $\beta$ must be a piece of a conic. Now by using a similar argument for the border points of $\beta$ and using regularity and simply connectedness of $M^{2}$, we can conclude that $M^{2}$ must be a ellipsoid of revolution.

Now, let us assume that $M^{2}$ is of genus one. Let $\mathscr{L}$ and $\mathscr{M}$ be homotopy classes corresponding to a latitude and a meridian respectively. Then $\mathscr{L}$ and $\mathscr{M}$ generate the fundamental group $\pi_{1}\left(M^{2}\right)$ of $M^{2}$. It is known [11] that an ellipse contained in $M^{2}$ must be in one of the following homotopy classes $\mathcal{O}, \mathscr{L}, \mathscr{M}$, $\mathscr{L}+\mathscr{M}$ or $\mathscr{L}-\mathscr{M}$, where $\mathcal{O}$ is the trivial homotopy class. We wish to prove that $M^{2}$ satisfies the following property:

(*) There exists a point $p \in M^{2}$ such that one of the ellipses through $p$ which is not a latitude, does not belong to $\mathcal{O} \cup \mathscr{L}$.

In fact, take $p_{0} \in M^{2}$ and suppose it is not a point satisfying (*), and consider an ellipse $E_{0}$ through $p_{0}$ which belongs to $\mathcal{O} \cup \mathscr{L}$. Then through almost every point 
of $E_{0}$ there passes a latitude $C_{0}$ which cuts $E_{0}$ in two different points. By using an argument analogous to that of the simply connected case, we can see that there is a closed piece of a conic $\beta$ which is included in the copy of the generating curve of $M^{2}, \gamma$, which passes through $p_{0}$. Take now a point $p_{1}$ of the border of $\beta$. If $M^{2}$ satisfies $(*)$ at $p_{1}$, we have done. If not, we obtain for $p_{1}$ the same conclusion as for $p_{0}$ and, therefore, by regularity, there exists a closed piece of a conic, which we also denote by $\beta$, that passes through $p_{0}$ and $p_{1}$ and is contained in $\gamma$. By repeating the process we see that either exists a point where $M^{2}$ satisfies (*) or $\gamma$ must be an ellipse through $p_{0}$, but the latter contradicts the election of $p_{0}$. This shows $(*)$. Then there exists an ellipse $\tilde{E}$ which belongs to one of $\mathscr{M}$, $\mathscr{L}+\mathscr{M}$ or $\mathscr{L}-\mathscr{M}$. This means that $M^{2}$ is swept by $\tilde{E}$ under rotation.

Q.E.D.

Now, suppose that we have an elliptic hulahoop surface $H(a, b, r, s, \beta, \alpha)$ (satisfying some of the regularity conditions (i) to (iv)). If we put

$$
\begin{aligned}
q(\theta)= & (a+r \cos \beta \cos \theta-s \sin \beta \sin \theta)^{2} \\
& +(b+r \sin \beta \cos \alpha \cos \theta+s \cos \beta \cos \alpha \sin \theta)^{2}
\end{aligned}
$$

then we observe that $H(a, b, r, s, \beta, \alpha)$ is obtained by revolving the curve of the $x z$-plane given by

$$
\left\{\begin{array}{l}
x=(q(\theta))^{1 / 2} \\
y=0 \\
z=r \sin \beta \sin \alpha \cos \theta+s \cos \beta \sin \alpha \sin \theta
\end{array}\right.
$$

with $\theta \in[0,2 \pi)$.

For a surface of revolution parametrized by

$$
x=(u(\theta) \cos \phi, u(\theta) \sin \phi, v(\theta))
$$

one can check by a straight-forward computation that the Laplace's operator is given by

$$
\Delta=-\frac{1}{h} \frac{\partial^{2}}{\partial \theta^{2}}-\left\{\frac{u^{\prime}}{u h}-\frac{u^{\prime} u^{\prime \prime}+v^{\prime} v^{\prime \prime}}{h^{2}}\right\} \frac{\partial}{\partial \theta}-\frac{1}{u^{2}} \frac{\partial^{2}}{\partial \phi^{2}}
$$

where $h=\left(u^{\prime}\right)^{2}+\left(v^{\prime}\right)^{2}$.

By using (6) for the parametrization given in (4), one can see that the Laplacian of $H(a, b, r, s, \beta, \alpha)$ for an $f \in C^{\infty}(H(a, b, r, s, \beta, \alpha))$ which depends only on $\theta$ satisfies

$$
\Delta f=-\frac{q}{g} f^{\prime \prime}-\left(\frac{q^{\prime}}{g}-\frac{g^{\prime} q}{2 g^{2}}\right) f^{\prime}
$$

where $g$ denote the discriminant of the first fundamental form. 
Proof of Proposition 2. We do our discusion in terms of the regularity conditions (i) to (iv). We first consider the case $r \neq s$, then $g(\theta)$ can be expressed as

$$
\begin{aligned}
g(\theta)= & \frac{1}{8}\left(r^{2}-s^{2}\right)\left(\cos ^{2} \beta\left(s^{2} \cos ^{2} \alpha-r^{2}\right)+\sin ^{2} \beta\left(s^{2}-r^{2} \cos ^{2} \alpha\right)\right) \cos 4 \theta \\
& +\frac{1}{4}\left(r^{2}-s^{2}\right) r s \sin \beta \cos \beta \sin ^{2} \alpha \sin 4 \theta \\
& -\frac{1}{2}\left(r^{2}-s^{2}\right) r(a \cos \beta+b \sin \beta \cos \alpha) \cos 3 \theta \\
& -\frac{1}{2}\left(r^{2}-s^{2}\right) s(-a \sin \beta+b \cos \beta \cos \alpha) \sin 3 \theta \\
& +\cdots
\end{aligned}
$$

Considering $g$ and $q$ as polynomials in $\cos \theta, \sin \theta$, we observe that $1 \leq$ $\operatorname{deg}(q) \leq 2$ and that $\operatorname{deg}(q)=1$, if and only if, $\beta=0$ and $r=s \cos \alpha$, where $\operatorname{deg}(q)$ means degree of $q$. Also we have $\operatorname{deg}(g)=\operatorname{deg}(q)+2$.

One can obtain by induction the following formula for the higher order Laplacian of the function $z$ given in (4)

$$
\Delta^{k} z=\frac{A_{k} \cos m \theta+B_{k} \sin m \theta+p_{k}}{g^{3 k-1}} \quad k \geq 0
$$

where $A_{k}, B_{k} \in \boldsymbol{R}$ do not vanish simultaneously and $p_{k}(\sin \theta, \cos \theta)$ is a polynomial of degree at most $m(k)-1$ with

$$
m(k)=(3 \operatorname{deg}(q)+4) k-(\operatorname{deg}(q)+1) .
$$

If $H(a, b, r, s, \beta, \alpha)$ were of finite Chen-type, we might use Proposition 3, (4) and (9) to obtain that for some $k \in \boldsymbol{N}$

$$
\tilde{p}_{k}+c_{k-1} \tilde{p}_{k-1} g^{3}+\cdots+c_{l} \tilde{p}_{l} g^{3(k-l)}+\cdots+c_{0}\left(z-z_{0}\right) g^{3 k-1}=0
$$

where $c_{j} \in R, \tilde{p}_{l}(\sin \theta, \cos \theta)$ is a polynomial of degree $m(l)$ and therefore $\tilde{p}_{l} g^{3(k-l)}$ is a polynomial of degree $(3 \operatorname{deg}(q)+6) k-(\operatorname{deg}(q)+1)-2 l, l=0, \ldots, k$.

Thus the coefficients $c_{j}$ must vanish for $j=0, \ldots, k-1$, which contradicts the assumption on the finiteness of the Chen-type.

Let us assume now $r=s$ and $a \neq 0$. Without loss of generality we can take $\beta=0$. In this case

$$
\begin{aligned}
g(\theta)= & \frac{q^{\prime}}{4}+4 r^{2} \sin ^{2} \alpha \cos ^{2} \theta q \\
= & 2 r^{2}\left(b^{2}+r^{2} \sin ^{2} \alpha-a^{2} \cos ^{2} \alpha\right) \cos 2 \theta \\
& -4 a b r^{2} \cos \alpha \sin ^{2} \theta+8 a r^{3} \sin ^{2} \alpha \cos \theta \\
& +2 a^{2} r^{2}\left(1+\sin ^{2} \alpha\right)+2 b^{2} r^{2}+2 r^{4} \sin ^{2} \alpha
\end{aligned}
$$

then $\operatorname{deg}(q)=2$ and $1 \leq \operatorname{deg}(g) \leq 2$ with $\operatorname{deg}(g)=1$, if and only if, $b=0$ and $r^{2} \sin ^{2} \alpha=a^{2} \cos ^{2} \alpha$. 
Again from (4), (7) and by induction one can check after a long computation

$$
\Delta^{k} z=a_{k} \frac{q^{\prime} q^{k}\left(4 g^{\prime}\right)^{2 k-2}}{(4 g)^{3 k-1}}+\frac{p_{k}}{(4 g)^{3 k-2}}
$$

where ()$^{\prime}$ is meant for the derivative with respect to $\theta$,

$$
a_{k}=\left(-2^{3} r^{2} a \sin \alpha\right)(-1)^{k-1} \cdot 1 \cdot 4 \cdot 7 \cdot \ldots \cdot(1+6(k-1))
$$

and $p_{k}$ is a polynomial in $\sin \theta, \cos \theta$ of degree $2 k+1+(2 k-2) \operatorname{deg}(g), k \geq 1$.

If $H(a, b, r, s, \beta, \alpha)$ were of finite Chen-type $k$, then by using Proposition 3, (4) and (11) we would obtain

$$
\frac{q^{\prime} q^{k}\left(4 g^{\prime}\right)^{2 k-2}}{4 g}=p(\sin \theta, \cos \theta)
$$

with $p(\sin \theta, \cos \theta)$ a polynomial in $\sin \theta, \cos \theta$. Consider the monic complex polynomials $P_{q}, P_{q^{\prime}}, P_{4 g}, P_{4 g^{\prime}}, P_{p}$ determined by the following relations

$$
\left\{\begin{array}{l}
\left(e^{i \theta}\right)^{2} q(\theta)=\varepsilon_{1} P_{q}\left(e^{i \theta}\right), \\
\left(e^{i \theta}\right)^{2} q^{\prime}(\theta)=\varepsilon_{2} P_{q^{\prime}}\left(e^{i \theta}\right), \\
4\left(e^{i \theta}\right)^{\operatorname{deg}(g)} g(\theta)=\varepsilon_{3} P_{4 g}\left(e^{i \theta}\right), \\
4\left(e^{i \theta}\right)^{\operatorname{deg}}(g) g^{\prime}(\theta)=\varepsilon_{4} P_{4 g^{\prime}}\left(e^{i \theta}\right), \\
\left(e^{i \theta}\right)^{m} p(\theta)=\varepsilon_{5} P_{p}\left(e^{i \theta}\right),
\end{array}\right.
$$

where $m=2(1+\operatorname{deg}(g)) k+2-3 \operatorname{deg}(g)$ and $\varepsilon_{i} \in C$. It is obvious that $\varepsilon_{2}=2 i \varepsilon_{1}$ and $\varepsilon_{4}=\operatorname{deg}(g) i \varepsilon_{3}$. Then from (12) we have

$$
\frac{P_{q^{\prime}}\left(P_{q}\right)^{k}\left(P_{4 g^{\prime}}\right)^{2 k-2}}{P_{4 g}}=P_{p} .
$$

If one observes $\operatorname{deg}\left(P_{4 g}\right)=\operatorname{deg}\left(P_{4 g^{\prime}}\right)=2 \operatorname{deg}(g)$ from (12) we get

$$
-4 \varepsilon_{1}^{2} P_{q^{\prime}}^{2}+2 r^{2} \sin ^{2} \alpha \varepsilon_{1}\left(T^{2}+1\right) P_{q}(T)=\varepsilon_{3} T^{2} P_{4 g}(T)
$$

with $T=e^{2 i \theta}$. By differentiating $q(\theta)$ and $4 g(\theta)$ we have

$$
\begin{gathered}
2 P_{q^{\prime}}(T)=-2 P_{q}(T)+T P_{q}^{\prime}(T), \\
\operatorname{deg}(g) P_{4 g^{\prime}}(T)=-\operatorname{deg}(g) P_{4 g}(T)+T P_{4 g}^{\prime}(T) .
\end{gathered}
$$

From (14), (15), (16) and (17) we see that $P_{4 g}$ must have double roots only.

As we said before, $1 \leq \operatorname{deg}(g) \leq 2$. If $\operatorname{deg}(g)=2$, then as a consequence of what we have just said, we could write

$$
P_{4 g}(T)=\left(T-\omega_{1}\right)^{2}\left(T-\omega_{2}\right)^{2},
$$

$\omega_{1}, \omega_{2} \in C$. But by identifying the coefficients of both polynomials we see that 
this is impossible unless

$$
b=0 \text { and } a^{2}=r^{2} \sin ^{2} \alpha .
$$

This means that our $H(a, b, r, s, \beta, \alpha)$ is a sort of anchor ring and we might do similar computations to those of [5], to see that none of them are of finite Chen-type.

If $\operatorname{deg}(g)=1$, we use an analogous argument to see that the only possibilities for the parameters $a, b, r, s, \sin \alpha$ are not compatible with the regularity conditions (i) to (iv).

Finally we have that the only remaining case is $r=s$ and $a=0$. But then $H(a, b, r, s, \beta, \alpha)$ is a round sphere which is of Chen-type 1 .

Q.E.D.

\title{
REFERENCES
}

[1] R. BLum, Circles on surfaces in the Euclidean 3-space, Lecture Notes in Math., 792, Springer, 1980, 213-221.

[2] T. E. CECIL AND P. Ryan, Tight and Taut Immersions of Manifolds, Research Notes in Math., 107, Pitman, 1985.

[3] B. Y. ChEN, Total Mean Curvature and Submanifolds of Finite Type, World Scientific Publisher, 1984.

[4] B. Y. CHEN, A report on submanifolds of finite type, Soochow J. Math., 22 (1996), 117-337.

[ 5] B. Y. CHEN, Surfaces of finite type in the Euclidean 3-space, Bull. Soc. Math. Belg. Ser B, 39 (1987), 243-254.

[6] B. Y. CHEN, Some open problems and conjectures on submanifolds of finite type, Soochow J. Math., 17 (1991), 169-188.

[7] B. Y. CHEN and S. IsHIKAWA, On classification of some surfaces of revolution of finite type, Tsukuba J. Math., 17 (1993), 287-298.

[8] O. J. GaraY, On a certain class of finite type surfaces of revolution, Kodai Math. J., 11 (1988), 25-31.

[9] T. Hasans and T. Vlachos, Surfaces of finite type with constant mean curvature, Kodai Math. J., 16 (1993), 244-252.

[10] K. Ogiue and N. Takeuchi, Hulahoop surfaces, J. Geom., 46 (1993), 127-132.

[11] D. Rolfsen, Knots and Links, Math. Lecture Ser., 7, Publish or Perish Inc., Berkeley, 1993.

\author{
Departamento de Matemáticas \\ Facultad de Ciencias \\ UNIVERSIDAD DEL País VASCo-Euskal HerRIKo Unibertsitatea \\ APARTADo 644, 48080 BILBAO \\ SPAIN \\ e-mail: mtparolj@lg.ehu.es \\ mtpgabeo@lg.ehu.es \\ mtpmegoj@lg.ehu.es
}

